\title{
Visusverlust nach dem Herzkatheter
}

— Eine 56-jährige Frau mit Hypertonie stellte sich am Tag nach einer Herzkatheteruntersuchung mit einem plötzlichen Visusverlust am rechten Auge vor. Die Katheteruntersuchung erfolgte im Rahmen einer hypertensiven Krise mit Angina pectoris und einem Blutdruck von 235/110 $\mathrm{mmHg}$. Die Sehschärfe am rechten Auge betrug 20/100 mit wesentlichen Gesichtsfeldeinschränkungen im unteren temporalen Quadranten.

Bei der Biomikroskopie erkannte man mehrere Cholesterinemboli (Abb. A, Pfeile) mit einem umgebenden Netzhautödem. Die Fluoreszenzangiografie bestätigte die Verschlüsse einer zilioretinalen Arterie und eine Ischämie im entsprechenden Versorgungsgebiet (Abb. B). Cholesterinemboli sind Kristalle, die aus ulzerierten und rupturierten arteriosklerotischen Plaques in die Zirkulation gelangen und initial an eine arterielle Verschlusskrankheit denken lassen.

Derartige embolische Ereignisse treten als seltene Komplikationen im Rahmen von Herzkatheteruntersuchungen auf und sind wahrscheinlich durch die Manipulation mit dem Katheter bedingt.

Die Patientin wurde mit isovolemischer Hämodilution und Heparin im Perfusor über fünf Tage hinweg behandelt. Nach drei Monaten hatte sich der Visus auf dem rechten Auge von 20/100 auf 20/60 verbessert.

H. S. FÜEßL =

\section{-C. H. Meyer, F. G. Holz}

(University of Bonn, Bonn, Germany, e-mail: meyer_eye@yahoo.com. Blurred vision after cardiac catheterization. New Engl. J. Med. 361 (2009) 24, 2366
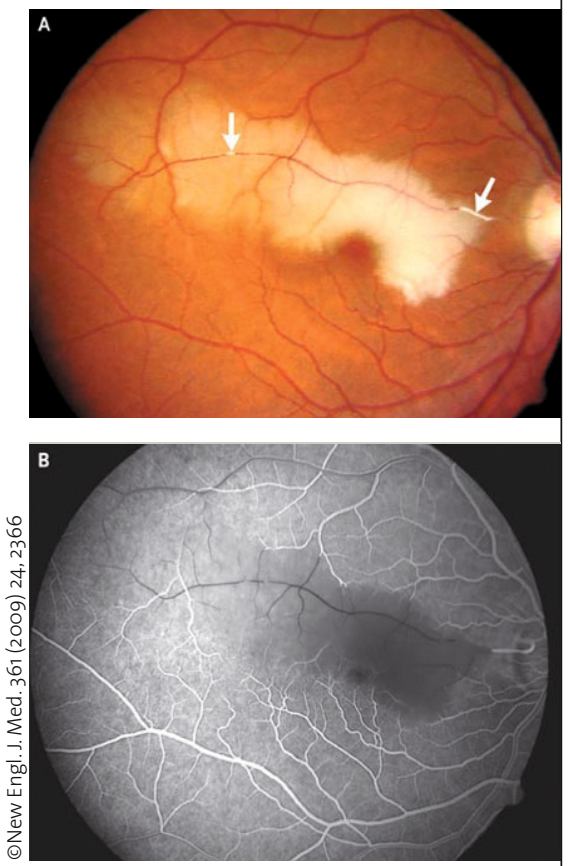

Cholesterinemboli (A, Pfeile) und Ischämie in der Netzhaut (B).

\section{Bisphosphonate erhöhen das gastrointestinale Blutungsrisiko nicht}

\section{Ob Bisphosphonate das Risiko für eine Magen-Darm-Blutung erhöhen, wird seit einigen Jahren kontrovers diskutiert. Eine neuere Studie gibt Entwarnung.}

— Im Rahmen dieser Fallkontrollstudie wurde das Risiko einer oberen gastrointestinalen Blutung bei Patienten untersucht, die entweder nur ein Bisphosphonat oder nur ein nicht steroidales Antiphlogistikum oder eine Kombination beider Substanzgruppen einnahmen. Nach entsprechender Risikoadjustierung fand sich für die Einnahme von Bisphosphonaten kein erhöhtes Blutungsrisiko. Die Einnahme eines NSAR erhöhte dagegen das Risiko für eine obere gastrointestinale Blutung um das 1,75-Fache. Bei gleichzeitiger Einnahme von Bisphosphonaten und NSAR fand sich nur eine minimale weitere Risikozunahme, d.h. das Risiko war zweifach erhöht, auch dann, wenn eine Cox-2-Inhibitor eingesetzt wurde.

\section{Kommentar}

Die Ergebnisse dieser Studie stehen in einem gewissen Widerspruch zu vorausgegangenen Berichten über gastrointestinale Schleimhautschäden und auch Magenulzera unter einer oralen Therapie mit einem Bisphosphonat. Da diese neue Fallkontrollstudie kein erhöhtes Risiko für eine obere gastrointestinale Blutung nachweisen konnte, besteht auch keine
Indikation für eine medikamentöse Prophylaxe mit einem Protonenpumpenhemmer bei einer Bisphosphonattherapie. Auch bei gleichzeitiger Gabe eines Bisphosphonats mit einem NSAR wird das Blutungsrisiko allein durch das nicht steroidale Antiphlogistikum bestimmt. Letzteres erfordert eine PPI-Prophylaxe insbesondere bei älteren Patienten, Patienten mit vorausgegangener Magenblutung und Patienten mit gleichzeitiger Einnahme eines Steroids oder von Marcumar.

P. StIEFELHAGEN =

\footnotetext{
- M. Etminan et al.

Risk of upper gastrointestinal bleeding with oral bisphosphonates and non-steroidal antiinflammatory drugs: A case-control study. Aliment. Pharmacol.Ther.29 (2009) 1188-1192
} 